\title{
AN OPTIMAL CONTROL PROBLEM FOR A FOURTH-ORDER VARIATIONAL INEQUALITY
}

\author{
A. M. KHLUDNEV
}

Lavrent'ev Institute of Hydrodynamics, Siberian Branch of the Russian Academy of Sciences

Novosibirsk 630090, Russia

An optimal control problem is considered where the state of the system is described by a variational inequality for the operator $w \rightarrow \varepsilon \Delta^{2} w-\varphi\left(\|\nabla w\|^{2}\right) \Delta w$. A set of nonnegative functions $\varphi$ is used as a control region. The problem is shown to have a solution for every fixed $\varepsilon>0$. Moreover, the solvability of the limit optimal control problem corresponding to $\varepsilon=0$ is proved. A compactness property of the solutions of the optimal control problems for $\varepsilon>0$ and their relation with the limit problem are established. This type of operator arises in the theory of nonlinear plates, and the choice of a most suitable function $\varphi$ is of interest for applications [2]. The problem of control of the function $w$ has been studied in [4] for the operator under consideration, and some statements of this work will be used. Nonstationary problems with analogous operators were analyzed in $[6,7]$. Some general results on control of second-order variational inequalities can be found in [1]. The first section of this paper deals with the control problem for our fourth-order operator, the second considers a second-order operator, and the third studies the relationship between the solutions of the two problems.

I. Fourth-order operator. Let $\Omega \subset \mathbb{R}^{2}$ be a bounded domain with a smooth boundary $\partial \Omega$; let $H^{s}(\Omega)$ be the Sobolev space of functions having $s$ generalized derivatives square summable in $\Omega$. The closure of the smooth compactly supported functions in $\Omega$ in the $H^{s}(\Omega)$ norm is denoted by $H_{0}^{s}(\Omega)$. Let $\psi \in H^{2}(\Omega)$ be a given function, $\left.\psi\right|_{\partial \Omega}<0$. We define a convex and closed set in $H^{2,0}(\Omega) \equiv H^{2}(\Omega) \cap H_{0}^{1}(\Omega)$ as follows:

$$
K_{2}=\left\{w \in H^{2,0}(\Omega) \mid w(x) \geq \psi(x), x \in \Omega\right\} .
$$

Consider the variational inequality

$$
\begin{aligned}
w \in K_{2}, \quad \varepsilon(\Delta w, \Delta \bar{w}-\Delta w)+\varphi\left(\|\nabla w\|^{2}\right)(\nabla w, \nabla \bar{w}-\nabla w) & \\
& \geq(f, \bar{w}-w) \quad \forall \bar{w} \in K_{2} .
\end{aligned}
$$


Here $(\cdot, \cdot)$ is the scalar product in $L^{2}(\Omega)$. Assume that $f \in L^{2}(\Omega)$. Let $\Phi$ be a convex and closed subset of $H^{1}(0, \infty)$ consisting of nonnegative functions. The cost functional is

$$
E_{\varepsilon}(\varphi)=\left\|w(\varphi)-w_{0}\right\|+\|\varphi\|_{1}, \quad \varphi \in \Phi .
$$

Here $w(\varphi)$ is the solution of the variational inequality (1) corresponding to $\varphi$ (some conditions on $\varphi$ ensuring the existence and uniqueness of solutions to (1) are given below); $w_{0} \in L^{2}(\Omega)$ is a prescribed element; $\|\cdot\|_{s}$ is the norm in $H^{s}(\Omega)$ or in $H^{s}(0, \infty),\|\cdot\|_{0} \equiv\|\cdot\|$. The optimal control problem is to find $\varphi \in \Phi$ that

$$
E_{\varepsilon}(\varphi) \leq E_{\varepsilon}(\bar{\varphi}) \quad \forall \bar{\varphi} \in \Phi .
$$

At this stage $\varepsilon>0$ is assumed to be fixed. The dependence of the solutions on $\varepsilon$ will be discussed later.

First, we present a well-known statement without proof.

LEMma 1. Let $\varphi \in \Phi$ and suppose $\sqrt{s} \varphi(s)$ is a nondecreasing function of $s$. Then the operator $w \rightarrow-\varphi\left(\|\nabla w\|^{2}\right) \Delta w$ is monotone from $H_{0}^{1}(\Omega)$ into its dual.

This lemma is a particular case of a statement proved in [3]. Note that $H^{1}(0, \infty)$ functions are continuous in $[0, \infty)$ (see [5]).

Assume that for each $\varphi \in \Phi$, the function $\sqrt{s} \varphi(s)$ is nondecreasing. Set

$$
\Pi_{\varepsilon}^{\varphi}(w)=\frac{\varepsilon}{2}\|\Delta w\|^{2}+\frac{1}{2} \int_{0}^{\|\nabla w\|^{2}} \varphi(s) d s-(f, w),
$$

which allows inequality (1) to be written as follows:

$$
w \in K_{2}, \quad \partial \Pi_{\varepsilon}^{\varphi}(w)(\bar{w}-w) \geq 0 \quad \forall \bar{w} \in K_{2} .
$$

Here $\partial \Pi_{\varepsilon}^{\varphi}(w)$ is the derivative of the functional $\Pi_{\varepsilon}^{\varphi}$ at the point $w$. Observe that, according to Lemma 1 , the operator $w \rightarrow \partial \Pi_{\varepsilon}^{\varphi}(w)$ is monotonous from $H^{2,0}(\Omega)$ into its dual, and therefore, the variational inequality (1) is equivalent to the problem of minimization of $\Pi_{\varepsilon}^{\varphi}(w)$ on $K_{2}$. It follows that, for every $\varphi \in \Phi,(1)$ has a unique solution. This is a consequence of the coercivity and lower semicontinuity of $\Pi_{\varepsilon}^{\varphi}$ on $H^{2,0}(\Omega)$.

THEOREM 1. Suppose $\Phi$ satisfies the above conditions. Then the optimal control problem (2) has a solution.

Proof. Choose a minimizing sequence $\varphi_{n} \in \Phi$. Then $\left\{\varphi_{n}\right\}$ is bounded in $H^{1}(0, \infty)$. Passing to a subsequence if necessary, we may assume that $\varphi_{n} \rightarrow \varphi$ weakly in $H^{1}(0, \infty)$. The problem

$$
w_{n} \in K_{2}, \quad \partial \Pi_{\varepsilon}^{\varphi_{n}}\left(w_{n}\right)\left(\bar{w}-w_{n}\right) \geq 0 \quad \forall \bar{w} \in K_{2}
$$

has a solution for every $n$. By fixing $\bar{w} \in K_{2}$, we may deduce from (3) that

$$
\Pi_{\varepsilon}^{\varphi_{n}}\left(w_{n}\right) \leq \Pi_{\varepsilon}^{\varphi_{n}}(\bar{w}) \leq c
$$


with a constant $c$ independent of $n$. Since $\varphi_{n} \geq 0$ we get

$$
\left\|\Delta w_{n}\right\|^{2} \leq c .
$$

Recall that so far $\varepsilon$ is considered to be fixed. The obtained estimate means that $\left\{w_{n}\right\}$ is bounded in $H^{2,0}(\Omega)$. Passing to a subsequence if necessary, we can assume that $w_{n} \rightarrow w$ weakly in $H^{2,0}(\Omega)$ and strongly in $H_{0}^{1}(\Omega)$. Let, moreover, $\left\|\nabla w_{n}\right\|^{2} \leq$ $\alpha$. Then, in addition, we may assume that $\varphi_{n} \rightarrow \varphi$ uniformly in $[0, \alpha]$. The latter follows from the compactness of the imbedding of $H^{1}(0, \alpha)$ in $C[0, \alpha]$. Now we can pass to the limit in (3) using the above-mentioned convergence. Indeed,

$$
\varphi_{n}\left(\left\|\nabla w_{n}\right\|^{2}\right) \rightarrow \varphi\left(\|\nabla w\|^{2}\right), \quad \liminf \left\|\Delta w_{n}\right\|^{2} \geq\|\Delta w\|^{2} .
$$

Therefore, the limit function $w$ satisfies

$$
w \in K_{2}, \quad \partial \Pi_{\varepsilon}^{\varphi}(w)(\bar{w}-w) \geq 0 \quad \forall \bar{w} \in K_{2},
$$

and hence $w=w(\varphi)$. The lower semicontinuity of the norm gives

$$
\inf _{\bar{\varphi} \in \Phi} E_{\varepsilon}(\bar{\varphi})=\liminf _{n \rightarrow \infty} E_{\varepsilon}\left(\varphi_{n}\right) \geq E_{\varepsilon}(\varphi) \geq \inf _{\varphi \in \Phi} E_{\varepsilon}(\bar{\varphi}) .
$$

This means that $\varphi$ minimizes $E_{\varepsilon}$ on $\Phi$. The proof is complete.

2. Second-order operator. Let us introduce a convex and closed set in $H_{0}^{1}(\Omega)$ by

and consider the variational inequality

$$
K_{1}=\left\{w \in H_{0}^{1}(\Omega) \mid w(x) \geq \psi(x), x \in \Omega\right\}
$$

$$
w \in K_{1}, \quad \varphi\left(\|\nabla w\|^{2}\right)(\nabla w, \nabla \bar{w}-\nabla w) \geq(f, \bar{w}-w) \quad \forall \bar{w} \in K_{1} .
$$

We assume that $\sqrt{s} \varphi(s)$ is strictly increasing for each $\varphi \in \Phi$. Moreover, we assume $\sqrt{s} \varphi(s) \rightarrow \infty$ as $s \rightarrow \infty$, uniformly in $\varphi \in \Phi$. Then for each fixed $\varphi \in \Phi$ there exists a unique solution of (5) (see [4]). The problem of minimization of the functional $\Pi_{0}^{\varphi}$ on $K_{1}$ is equivalent to the variational inequality (5), analogously to $(1)$.

Now consider the optimal control problem with the same cost functional:

$$
E_{0}(\varphi)=\left\|w(\varphi)-w_{0}\right\|+\|\varphi\|_{1}
$$

where $w(\varphi)$ is the solution of (5). An element $\varphi \in \Phi$ is to be found so that

$$
E_{0}(\varphi) \leq E_{0}(\bar{\varphi}) \quad \forall \bar{\varphi} \in \Phi .
$$

THEOREM 2. Under the above conditions on $\Phi$, the optimal control problem (6) has a solution.

Pro of. Let $\varphi_{n} \in \Phi$ be a minimizing sequence. Without loss of generality, we may assume that $\varphi_{n} \rightarrow \varphi$ weakly in $H^{1}(0, \infty)$. The variational inequality

$$
w_{n} \in K_{1}, \quad \varphi_{n}\left(\left\|\nabla w_{n}\right\|^{2}\right)\left(\nabla w_{n}, \nabla \bar{w}-\nabla w_{n}\right) \geq\left(f, \bar{w}-w_{n}\right) \quad \forall \bar{w} \in K_{1}
$$

has a solution for every $n$. An equivalent form of (7) is

$$
w_{n} \in K_{1}, \quad \Pi_{0}^{\varphi_{n}}\left(w_{n}\right) \leq \Pi_{0}^{\varphi_{n}}(\bar{w}) \quad \forall \bar{w} \in K_{1} .
$$


Let us show that $\Pi_{0}^{\varphi_{n}}\left(w_{n}\right)$ is coercive uniformly in $\varphi \in \Phi$. Indeed, we have

$$
\Pi_{0}^{\varphi}(w)-\Pi_{0}^{\varphi}(0)=\int_{0}^{1} \partial \Pi_{0}^{\varphi}(s w)(w) d s .
$$

Therefore,

$$
\begin{aligned}
\Pi_{0}^{\varphi}(w)= & \int_{0}^{1 / 2}\left(\partial \Pi_{0}^{\varphi}(s w)-\partial \Pi_{0}^{\varphi}(0)\right)(w) d s \\
& +\frac{1}{2} \partial \Pi_{0}^{\varphi}(0)(w)+\int_{1 / 2}^{1} \partial \Pi_{0}^{\varphi}(s w)(w) d s .
\end{aligned}
$$

According to Lemma 1, the first term of the right-hand side is non-negative; the second is equal to $-\frac{1}{2}(f, w)$, and the third is $\partial \Pi_{0}^{\varphi}(\bar{s} w)(w), \bar{s} \in[1 / 2,1]$. Consequently,

$$
\begin{aligned}
\Pi_{0}^{\varphi}(w) & \geq \frac{1}{2} \partial \Pi_{0}^{\varphi}(\bar{s} w)(w)-\frac{1}{2}(f, w) \\
& \geq \frac{1}{2}\|\nabla w\|\left(\varphi\left(\|\nabla w\|^{2} \bar{s}^{2}\right)\|\bar{s} \nabla w\|-c\right) \rightarrow \infty
\end{aligned}
$$

as $\|\nabla w\| \rightarrow \infty$, uniformly in $\varphi \in \Phi$. Fixing $\bar{w}$ in (8), we may assume that $\Pi_{0}^{\varphi_{n}}\left(w_{n}\right) \leq c$ with a constant $c$ independent of $n$. By the coercivity of $\Pi_{0}^{\varphi}$, we conclude that there exists a constant $c$ independent of $n$ such that

$$
\left\|\nabla w_{n}\right\|^{2} \leq c .
$$

As previously, we can assume additionally that $\varphi_{n} \rightarrow \varphi$ strongly in $C[0, \alpha]$. Let also $w_{n} \rightarrow w$ weakly in $H_{0}^{1}(\Omega)$. Note that $w \in K_{1}$. Now we wish to pass to the limit in (7). Inequality (8), equivalent to (7), takes the form

$$
\frac{1}{2} \int_{0}^{\left\|\nabla w_{n}\right\|^{2}} \varphi_{n}(s) d s-\left(f, w_{n}\right) \leq \frac{1}{2} \int_{0}^{\left\|\nabla \bar{w}_{n}\right\|^{2}} \varphi_{n}(s) d s-(f, \bar{w}) .
$$

At the same time, by the above considerations,

$$
\liminf \int_{0}^{\left\|\nabla w_{n}\right\|^{2}} \varphi_{n}(s) d s \geq \int_{0}^{\|\nabla w\|^{2}} \varphi(s) d s .
$$

Thus, after passing to the lower limit in both sides of (8), we obtain

$$
\Pi_{0}^{\varphi}(w) \leq \Pi_{0}^{\varphi}(\bar{w}) \quad \forall \bar{w} \in K_{1},
$$

which is equivalent to

$$
w \in K_{1}, \quad \varphi\left(\|\nabla w\|^{2}\right)(\nabla w, \nabla \bar{w}-\nabla w) \geq(f, \bar{w}-w) \quad \forall \bar{w} \in K_{1} .
$$

This means that $w=w(\varphi)$. The proof is completed as in Theorem 1 .

3. On the relationship between the solutions as $\varepsilon \rightarrow 0$. We assume the same conditions on $\varphi \in \Phi$ as in the previous section. We need the following 
statement on the approximation of a function satisfying a bound of the form $\bar{w} \geq \psi$ by a sequence of more smooth functions [4].

Lemma 2. For every $\bar{w} \in K_{1}$ there exists a sequence $\bar{w}^{n} \in K_{2}$ strongly converging to $\bar{w}$ in $H_{0}^{1}(\Omega)$.

Let $\varphi_{\varepsilon}$ be a solution of problem (2), and let $w\left(\varphi_{\varepsilon}\right)$ be the corresponding solution of the variational inequality (1). The relation between the solutions of the optimal control problems (2) and (6) is characterized by the following statement.

Theorem 3. Passing to subsequences if necessary, we have

$$
\begin{aligned}
\varphi_{\varepsilon} & \rightarrow \varphi \quad \text { weakly in } H^{1}(0, \infty), \\
w\left(\varphi_{\varepsilon}\right) & \rightarrow w \quad \text { weakly in } H_{0}^{1}(\Omega), \\
E_{\varepsilon}\left(\varphi_{\varepsilon}\right) & \rightarrow E_{0}(\varphi) .
\end{aligned}
$$

Here $\varphi$ is a solution of problem (6), and $w$ is the solution of (5) corresponding to $\varphi$.

Proof. Let $\varphi \in \Phi$ be any fixed element. Then for every $\varepsilon$

$$
E_{\varepsilon}\left(\varphi_{\varepsilon}\right) \leq E_{\varepsilon}(\varphi) .
$$

Let us show that the solutions $w(\varphi) \equiv w_{\varepsilon}(\varphi)$ of the variational inequality (1) corresponding to $\varphi$ have $H^{1}$ norms bounded uniformly in $\varepsilon$. This means, in particular, the boundedness of the right-hand side of (9). The variational inequality

$$
w_{\varepsilon}(\varphi) \in K_{2}, \quad \partial \Pi_{\varepsilon}^{\varphi}\left(w_{\varepsilon}(\varphi)\right)\left(\bar{w}-w_{\varepsilon}(\varphi)\right) \geq 0 \quad \forall \bar{w} \in K_{2},
$$

is equivalent to

$$
\Pi_{\varepsilon}^{\varphi}\left(w_{\varepsilon}(\varphi)\right) \leq \Pi_{\varepsilon}^{\varphi}(\bar{w}) \quad \forall \bar{w} \in K_{2} .
$$

Hence, for all $\varepsilon$,

$$
\frac{\varepsilon}{2}\left\|\Delta w_{\varepsilon}(\varphi)\right\|^{2}+\frac{1}{2} \int_{0}^{\left\|\nabla w_{\varepsilon}(\varphi)\right\|^{2}} \varphi(s) d s-\left(f, w_{\varepsilon}(\varphi)\right) \leq c,
$$

and thus

with $\alpha$ independent of $\varepsilon$

$$
\left\|\nabla w_{\varepsilon}(\varphi)\right\|^{2} \leq \alpha
$$

Therefore, $E_{\varepsilon}(\varphi)$ is bounded uniformly in $\varepsilon$, and then from (9) it follows that

$$
\left\|\varphi_{\varepsilon}\right\|_{1} \leq c .
$$

Passing to a subsequence if necessary, we may assume that $\varphi_{\varepsilon} \rightarrow \varphi$ weakly in $H^{1}(0, \infty)$. Then from the inequality

$$
w_{\varepsilon}\left(\varphi_{\varepsilon}\right) \in K_{2}, \quad \partial \Pi_{\varepsilon}^{\varphi_{\varepsilon}}\left(w_{\varepsilon}\left(\varphi_{\varepsilon}\right)\right)\left(\bar{w}-w_{\varepsilon}\left(\varphi_{\varepsilon}\right)\right) \geq 0 \quad \forall \bar{w} \in K_{2}
$$

we get an estimate for $w_{\varepsilon}\left(\varphi_{\varepsilon}\right)$. Indeed, (10) is equivalent to

$$
\Pi_{\varepsilon}^{\varphi_{\varepsilon}}\left(w_{\varepsilon}\left(\varphi_{\varepsilon}\right)\right) \leq \Pi_{\varepsilon}^{\varphi_{\varepsilon}}(\bar{w}) \quad \forall \bar{w} \in K_{2} .
$$


So

$$
\varepsilon\left\|\Delta w_{\varepsilon}\left(\varphi_{\varepsilon}\right)\right\|^{2}+\left\|\nabla w_{\varepsilon}\left(\varphi_{\varepsilon}\right)\right\| \leq \sqrt{\alpha}
$$

with some constant $\alpha$ independent of $\varepsilon$. Taking a subsequence if necessary we may assume that, as $\varepsilon \rightarrow 0$,

$$
\begin{aligned}
& w_{\varepsilon}\left(\varphi_{\varepsilon}\right) \rightarrow w \quad \text { weakly in } H_{0}^{1}(\Omega), w \in K_{1}, \\
& \varepsilon w_{\varepsilon}\left(\varphi_{\varepsilon}\right) \rightarrow 0 \quad \text { weakly in } H^{2,0}(\Omega) \text {. }
\end{aligned}
$$

Assume additionally that $\varphi_{\varepsilon} \rightarrow \varphi$ uniformly in $[0, \alpha]$. From (11) it follows that

$$
\frac{1}{2} \int_{0}^{\left\|\nabla w_{\varepsilon}\left(\varphi_{\varepsilon}\right)\right\|^{2}} \varphi_{\varepsilon}(s) d s-\left(f, w_{\varepsilon}\left(\varphi_{\varepsilon}\right)\right) \leq \Pi_{\varepsilon}^{\varphi_{\varepsilon}}(\bar{w}) .
$$

Letting $\varepsilon \rightarrow 0$ with fixed $\bar{w} \in K_{2}$ we have

$$
\frac{1}{2} \int_{0}^{\|\nabla w\|^{2}} \varphi(s) d s-(f, w) \leq \Pi_{0}^{\varphi}(\bar{w}) .
$$

By Lemma 2, we conclude that (12) is satisfied for every $\bar{w} \in K_{1}$. Therefore,

$$
\varphi\left(\|\nabla w\|^{2}\right)(\nabla w, \nabla \bar{w}-\nabla w) \geq(f, \bar{w}-w) \quad \forall \bar{w} \in K_{1} .
$$

This means that $w=w(\varphi)$ and, consequently,

$$
\liminf E_{\varepsilon}\left(\varphi_{\varepsilon}\right) \geq E_{0}(\varphi) .
$$

On the other hand, for any fixed $\varphi \in \Phi$, and possibly for a subsequence, $E_{\varepsilon}(\varphi) \rightarrow$ $E_{0}(\varphi)$. Indeed, from the variational inequality

$$
w_{\varepsilon}(\varphi) \in K_{2}, \quad \partial \Pi_{\varepsilon}^{\varphi}\left(w_{\varepsilon}(\varphi)\right)\left(\bar{w}-w_{\varepsilon}(\varphi)\right) \geq 0 \quad \forall \bar{w} \in K_{2}
$$

we get

$$
\left.\left.\varepsilon \| \Delta w_{\varepsilon}(\varphi)\right)\left\|^{2}+\right\| \nabla w_{\varepsilon}(\varphi)\right) \| \leq c
$$

uniformly in $\varepsilon$. Taking a subsequence if necessary, we may assume

$$
\begin{aligned}
w_{\varepsilon}(\varphi) & \rightarrow \widetilde{w} & & \text { weakly in } H_{0}^{1}(\Omega), \text { strongly in } L^{2}(\Omega), \\
\varepsilon w_{\varepsilon}(\varphi) & \rightarrow 0 & & \text { weakly in } H^{2,0}(\Omega) .
\end{aligned}
$$

Let $\varepsilon \rightarrow 0$ in (14), as in (10), to obtain

$$
\widetilde{w} \in K_{1}, \quad \varphi\left(\|\nabla \widetilde{w}\|^{2}\right)(\nabla \widetilde{w}, \nabla \bar{w}-\nabla \widetilde{w}) \geq(f, \bar{w}-\widetilde{w}) \quad \forall \bar{w} \in K_{1} .
$$

It follows that $\widetilde{w}=w(\varphi)$, so that

$$
E_{\varepsilon}(\varphi) \equiv\left\|w_{\varepsilon}(\varphi)-w_{0}\right\|+\|\varphi\|_{1} \rightarrow E_{0}(\varphi) .
$$

If now $\widetilde{\varphi}$ is a solution of the optimal control problem (6), we have

$$
E_{\varepsilon}\left(\varphi_{\varepsilon}\right) \leq E_{\varepsilon}(\widetilde{\varphi}) .
$$

Therefore

$$
\lim \sup E_{\varepsilon}\left(\varphi_{\varepsilon}\right) \leq E_{0}(\widetilde{\varphi}) .
$$

Together with (13), this concludes the proof. 


\section{References}

[1] V. Barbu, Optimal Control of Variational Inequalities, Res. Notes in Math. 100, Pitman, 1984.

[2] E. I. Grigolyuk and G. M. Kulikow, On a simplified method of solution of nonlinear problems in elastic plate and shell theory, in: Some Applied Problems of Plate and Shell Theory, Moscow University, 1981, 94-121 (in Russian).

[3] A. M. Khludnev, A boundary-value problem for a system of equations with a monotone operator, Differentsial'nye Uravneniya 16 (10) (1980), 1843-1849 (in Russian).

[4] - On limit passages in optimal control problems for a fourth-order operator, ibid. 25 (8) (1989), 1427-1435 (in Russian).

[5] J.-L. Lions and E. Magenes, Non-homogeneous Boundary Value Problems and Applications, Springer, 1972.

[6] S. I. Pokhozhaev, On a class of quasilinear hyperbolic equations, Mat. Sb. 96 (1) (1975), 152-166 (in Russian).

[7] -, On a quasilinear hyperbolic Kirchhoff equation, Differentsial'nye Uravneniya 21 (1) (1985), 101-108 (in Russian). 